\title{
Disponibilidad de médicos y especialistas en Chile
}

\author{
MICHÈLE GUILLOU ${ }^{a}$, JORGE CARABANTES C., VERÓNICA BUSTOS F. ${ }^{\text {b }}$
}

\section{Availability of physicians and specialists in Chile}

The availability and planning of Human Resources are important issues in many countries, as it is a key factor to cope with the critical challenges of Health Care Systems. In Chile, the Ministry of Health has undertaken several studies in order to improve knowledge about the medical workforce both in public and private sectors. The aim of this paper is to update and systematize the existing data on physicians and specialists availability in Chile. Several information sources were crossed to obtain new and more precise figures about this topic. According to the Internal Revenue System, 29.996 physicians practice medicine in the country, 43\% of them hired in public services, part or full time. There is a high concentration of professionals in the central regions of Chile. Being the overall density of physicians of one per 559 inhabitants, the figures in the central region is one per 471 and one per more than 800 in the South and North. Between 2004 and 2008, the public sector increased its physician workforce by more than $80 \%$ in primary health care and more than $20 \%$ in the secondary and tertiary levels. This paper presents a method for a more rigorous identification of the categories of general practitioner and specialist respectively, and the results obtained from the databases used.

(Rev Med Chile 2011; 139: 559-570).

Key words: Health planning guidelines; Health resources; Health Services research.

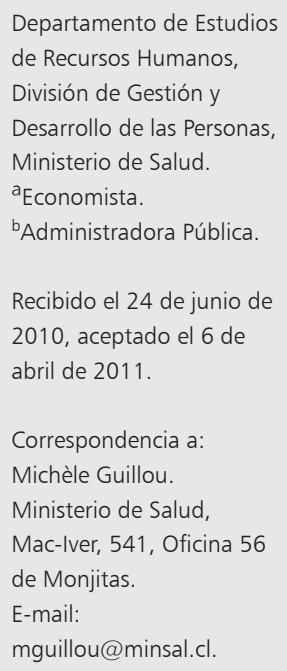

L a preocupación por disponer, desarrollar y retener una fuerza laboral acorde a las necesidades de salud y expectativas de la población, considerando su sustentabilidad económica, se encuentra en la agenda política de muchos países.

En varias partes del mundo se observan ciclos de escasez y sobreoferta de recursos humanos calificados, además de problemas de distribución geográfica, lo que genera iniciativas internacionales, tanto en Europa como en las Américas ${ }^{1-4}$.

En Chile, uno de los objetivos centrales del proceso de reforma sectorial es lograr el acceso universal a la salud, en una población que demanda cada vez más y mejores servicios de salud, lo que ha exigido un aumento de su complejidad y mayores requerimientos de recurso humano calificado en un sistema con dos mercados laborales (público y privado).

La demanda y oferta de médicos especialistas depende de múltiples factores, tales como el perfil demográfico y epidemiológico de la población, el modelo de atención, la evolución científica y tecnológica, la dinámica del mercado laboral, las expectativas de la ciudadanía, la judicialización en la salud, etc.; y de muchos actores ${ }^{5}$. También hay que considerar el efecto de la migración regional de profesionales.

El Ministerio de Salud ha desarrollado estudios para objetivar los problemas de disponibilidad de médicos especialistas ${ }^{6,7}$. El Gobierno de Chile solicitó al Banco Mundial la realización de un estudio que abordara los elementos determinantes de la disponibilidad de médicos especialistas, y propusiera cursos de acción en distintas áreas para enfrentar los déficits que se detectaran ${ }^{8}$.

La falta de fuentes de datos integrales que incluyan a la totalidad de los médicos del país ha dificultado su conocimiento, lo que se ha resuelto 
en buena medida utilizando la información de distintas bases.

El propósito de este artículo es actualizar y sistematizar la información existente sobre los médicos en Chile, particularmente los especialistas, analizar su distribución y características, condición previa indispensable a la instalación de un sistema de planificación de Recursos Humanos que cuente con la participación de los actores relevantes en la construcción de las soluciones a los problemas detectados.

\section{Material y Método}

El conocimiento actualizado de los médicos especialistas es complejo cuando no existe un registro nacional y porque la creación de nuevas especialidades es un proceso dinámico; además no siempre hay formalidad en su reconocimiento que cubra la totalidad de las situaciones. Por último, los médicos ejercen en distintos empleadores y modalidades, lo que dificulta la determinación de las horas requeridas para prestar atención $n^{9-11}$.

\section{Cuantificación del universo de los médicos y distribución geográfica}

El número total de médicos en Chile ha sido estimado a partir de la información provista por el Servicio de Impuestos Internos (SII), respecto a las personas naturales que pagan impuestos en actividades de servicios médicos. Quedan excluidas las empresas o sociedades de médicos. Dicho Servicio no entrega información que permita identificar a las personas, por lo que sólo se puede disponer del número de médicos por comuna, en las localidades que cuentan al menos con 10 profesionales.

La cifra de médicos que ejercen en el sector público se obtiene de los sistemas de información de Recursos Humanos (SIRH) de los Servicios de Salud (SS) y de la Atención Primaria Municipal (APS). Estas fuentes tienen un alto nivel de confiabilidad sobre los médicos con cargo de planta o contrata. A este segmento se debería agregar la información referida a consultores de llamada y contratos de servicios, cuyo número de convenios es de 1.581 al mes de abril de 2009 a nivel nacional (fuente: SIRH). Sin embargo, no fue posible precisar cuántos de dichos contratos corresponden a labores realizadas por profesionales funcionarios fuera de la jornada de trabajo habitual, evitando producir duplicidades de información.
La cantidad de médicos con actividad exclusiva en el sector privado se estimó por diferencia entre los registros del sector público y la información entregada por el Servicio de Impuestos Internos.

\section{Distribución por especialidad: Aspectos generales}

En Chile, se entiende por especialistas a aquellos profesionales que han tenido una formación de a lo menos tres años y que han sido titulados por una Universidad o certificados por una entidad reconocida. El sistema de certificación de especialistas, establecido en el Decreto Supremo No 57 de 2007, abordó la situación de los médicos que se desempeñan como especialistas sin tener el título universitario o certificación de la Corporación Nacional Autónoma de Certificación de Especialidades Médicas (Conacem). En él se establece un sistema definitivo que, de acuerdo al Decreto No 114 del 3 de noviembre de 2010, estará plenamente vigente a partir del $1^{\circ}$ de enero de 2013, y uno transitorio que le permite a un profesional médico que cumple con determinados requisitos de formación y experiencia, solicitar su inscripción como especialista en el registro de la Superintendencia de Salud. Esta inscripción estará vigente hasta noviembre de 2015 , fecha en que se deberán someter a un proceso de re-certificación periódico.

Las principales fuentes de información disponibles (SIRH, Conacem, Fondo Nacional de Salud (Fonasa) y Universidades) incorporan denominaciones de especialidades y subespecialidades heterogéneas, por lo que ha sido necesario:

- generar un mecanismo de estandarización y homologación de las denominaciones de las distintas nomenclaturas existentes, para implementar una designación única en el sistema de información de recursos humanos del Sector Público de Salud,

- y aplicar un procedimiento explícito de asignación de la especialidad a un profesional médico, cuando las fuentes difieren entre sí.

Para atender el primer requerimiento se homologaron denominaciones considerando la designación de las especialidades y subespecialidades incluidas en el Decreto Supremo No 57/2007, la utilizada por las Universidades, Conacem y el SIRH, y la referencia de la Oficina Internacional del Trabajo (OIT) $)^{12}$. 
Respecto de la segunda necesidad, se utilizaron las fuentes de Conacem, SIRH y APS, y del Ciclo de Destinación y Formación, las que incluyen:

- el RUT de cada persona, lo que evita duplicaciones,

- la comuna de localización del profesional.

Para confirmar la especialidad de un médico, se aplicó el procedimiento siguiente:

- En los contratados en un Servicio de Salud, la información dada por Conacem prevalece sobre la del SIRH.

- Para los demás médicos, la información entregada por Conacem prevalece sobre la del Ciclo de Destinación y de APS.

- Para ambos grupos, la subespecialidad prevalece sobre la especialidad.

En el Anexo 1, se entrega una descripción sucinta de los sistemas de información disponibles.

\section{Distribución por especialidad:}

\section{Estimación en el segmento privado}

La metodología y los procedimientos señalados permiten obtener una información homologada sobre la distribución por especialidad de los médicos contratados en el sector público. La información del Servicio de Impuestos Internos no indica la especialidad del contribuyente y no es individual. Por esto, se ha compuesto ${ }^{8}$ una base de datos nominalizada por RUT, sin duplicaciones, a través de cuatro fuentes de datos: SIRH, APS, Fonasa, Conacem; lográndose identificar 22.822 médicos de los 29.996 informados por el Servicio de Impuestos Internos. Los médicos que no se encuentran registrados en SIRH o APS se asumen como profesionales que ejercen exclusivamente en el sector privado sin perjuicio que pueden intervenir en el sector público a través de la venta de prestaciones. En el Anexo 2, se describe el proceso utilizado.

\section{Médicos generales y médicos especialistas}

Los análisis referidos a la identificación, caracterización y evolución de la disponibilidad de médicos en el Sector Público incluyen a todas las categorías de estos profesionales, tanto generalistas como especialistas. La Atención Primaria en Salud no es el foco de este artículo, pero los médicos contratados en ella están incorporados en los análisis globales. Lo señalado se aplica a los resultados presentados en los puntos 1 y 2, salvo indicación específica. Los resultados incluidos en el punto 3 se refieren sólo a los médicos especialistas.

\section{Resultados}

\section{Los médicos en Chile: Distribución geográfica y repartición entre el sector público y privado}

1.1. Según el Servicio de Impuestos Internos, el número de médicos en Chile se estima en 29.996 profesionales a diciembre de 2007, cifra superior a la

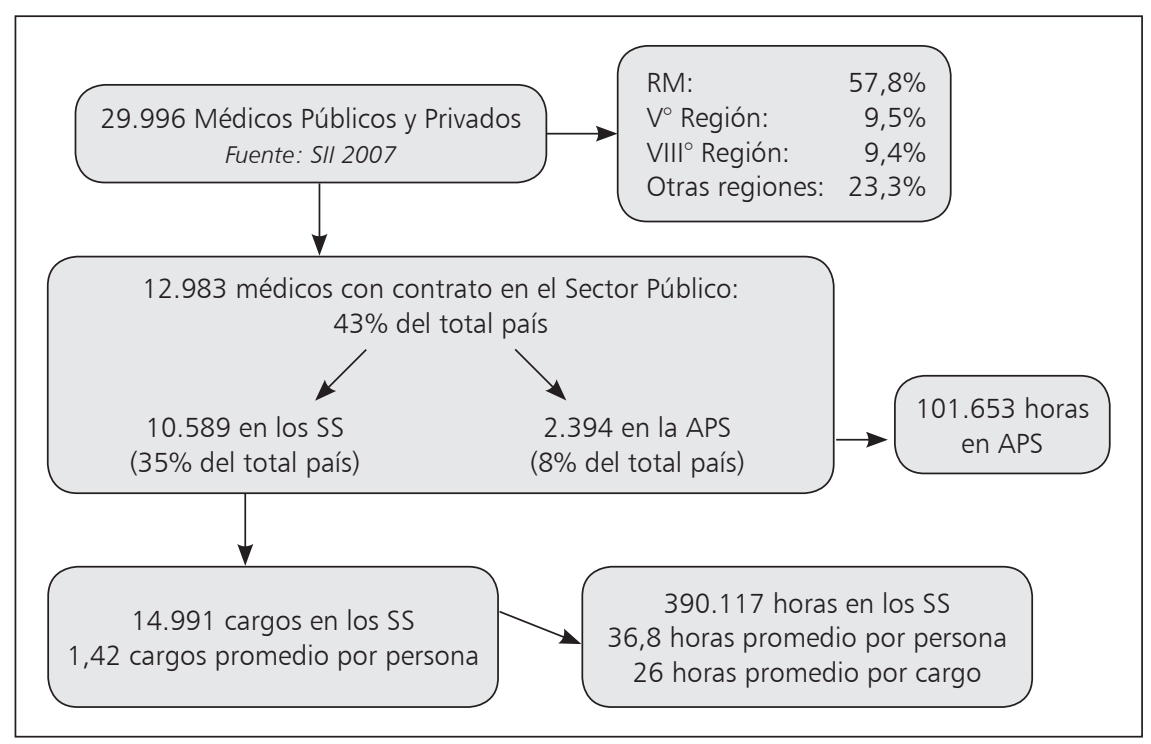

Figura 1. Estimación del universo de médicos en Chile. 
proyectada por Bastías y cols $(25.705)^{13}$. En la Figura 1 se observa la concentración en las Regiones Metropolitana (RM), Va y VIII a y la distribución entre el sector público y privado. Los profesionales identificados en el sector público son los que cuentan con al menos un cargo en un Servicio de Salud o en la Atención Primaria Municipal. Este segmento representa en 2008, 43\% de los profesionales médicos del país.

El número de horas disponibles se conoce sólo respecto del segmento público, por falta de información sobre el sector privado.

1.2. La Tabla 1 muestra que 73\% de los médicos se desempeña en la zona central del país (Regiones $V, V I$, VII $y$ XIII).

El número de médicos por 100.000 habitantes es de 179 a nivel nacional ( 310 en promedio en 30 países de la Organización para la Cooperación y el Desarrollo -OCDE- en 2005), mientras la tasa alcanza a 117 y 119 en las regiones sur y norte, respectivamente, y 212 en la central. El país tiene en promedio 1 médico por cada 559 habitantes. Este indicador es de 1 médico por 471 habitantes en la zona central y de 1 médico por 837 y 857 habitantes en las regiones Norte y Sur, respectivamente (Figura 2).

1.3. El número de beneficiarios por médico es de 276 en el segmento afiliado a una ISAPRE u otro seguro privado, y de 920 para los beneficiarios de FONASA (Figura 3). Parte de los médicos del Sistema Público comparte su tiempo entre sectores público y privado, en desmedro de la disponibilidad de horas para la población afiliada al Fonasa; a su vez, parte de ésta se atiende en la modalidad

Tabla 1. Distribución geográfica del total de los médicos en Chile

\begin{tabular}{|lcccc|}
\hline $\begin{array}{l}\text { Área } \\
\text { geográfica }\end{array}$ & $\begin{array}{c}\text { Cantidad } \\
\text { de médicos }\end{array}$ & $\%$ & $\begin{array}{c}\text { Médicos por área geográfica } \\
\mathbf{N}^{\circ} \text { de médicos por cada } \\
\mathbf{1 0 0 . 0 0 0} \text { habitantes }\end{array}$ & $\begin{array}{c}\mathbf{N}^{\circ} \text { de habitantes por } \\
\text { médico }\end{array}$ \\
Norte & 2.418 & 8,1 & 119,5 & 837,0 \\
Central & 21.899 & 73,0 & 212,1 & 471,4 \\
Centro Sur & 4.415 & 14,7 & 132,2 & 756,5 \\
Sur & 1.264 & 4,2 & 117,5 & 851,0 \\
\hline País & 29.996 & 100,0 & 178,9 & 558,9 \\
\hline
\end{tabular}

Fuente: Servicio de Impuestos Internos e INE (Población 2008). Aspectos metodológicos: El Área Norte agrupa a las regiones I, II, III,IV y XV; y el Área Central agrupa a las regiones V, VI, VII y XIII; el Área Centro-Sur agrupa a las regiones VIII, IX y XIV; y el Área Sur agrupa a las regiones X, XI y XII. Fuente datos de población: Instituto Nacional de Estadísticas (INE).

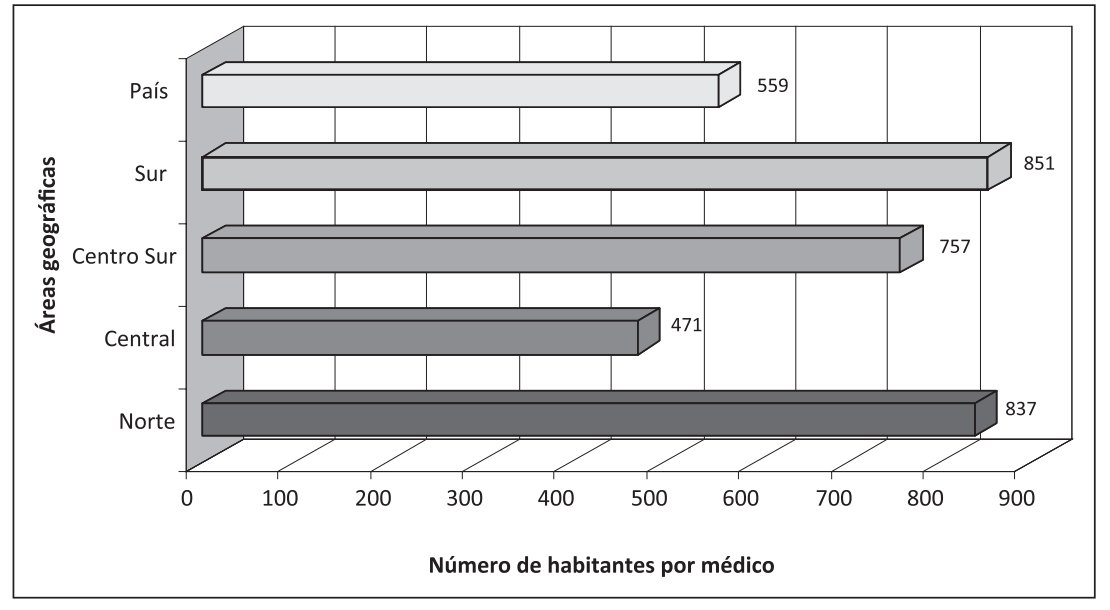

Figura 2. Número de habitantes por médico. Chile, 2008. Fuentes: Servicio de Impuestos Internos e INE (Población 2008). 


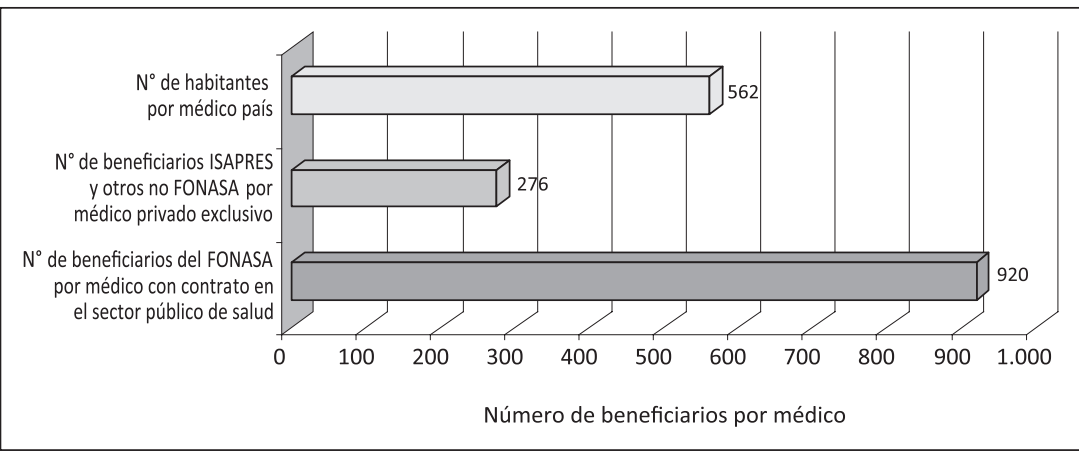

Figura 3. Número de beneficiarios por médico en Chile, según tipo de seguro. Año 2008. Fuentes: INE, SII, FONASA, SIRH, APS municipal. Médicos generales y especialistas. de libre elección. No se accedió a una fuente de información que entregara una cuantificación precisa sobre este aspecto.

\section{Los médicos en el Sector Público}

2.1. La evolución de la dotación de médicos entre los años 2004 y 2008, en el sector público, se puede apreciar en la Tabla 2, junto a la variación de la población beneficiaria.

El fortalecimiento de la Atención Primaria se expresa en el crecimiento sostenido de la disponibilidad de profesionales médicos, tanto en horas (+103,71\% entre 2004 y 2008) como en número de cargos $(+81 \%)$.

En los establecimientos de mayor complejidad, la dotación en cargos aumentó en casi $25 \%$ en el período en estudio, mientras que ha sido menor en número de profesionales y horas $(+20 \%$ y $+18 \%$ respectivamente). Esto refleja la tendencia creciente de los médicos de compar-

Tabla 2. Evolución disponibilidad de médicos en el sector público y población beneficiaria

\begin{tabular}{|lccccc|}
\hline & \multicolumn{5}{c}{ Evolución N $\mathbf{N}^{\circ}$ de médicos y población beneficiaria } \\
\hline Médicos servicios de salud - Cargos & $\mathbf{2 0 0 4}$ & $\mathbf{2 0 0 5}$ & $\mathbf{2 0 0 6}$ & $\mathbf{2 0 0 7}$ & $\mathbf{2 0 0 8}$ \\
\hline Médicos servicios de salud - Personas & 12.054 & 13.263 & 13.714 & 14.278 & 14.991 \\
\hline Médicos servicios de salud - Horas & 8.812 & 9.536 & 9.744 & 10.120 & 10.589 \\
\hline Médicos APS municipal - Personas & 330.625 & 353.998 & 361.229 & 373.094 & 390.117 \\
\hline Médicos APS municipal - Horas & 1.495 & 1.745 & 1.921 & 2.512 & 2.748 \\
\hline Población beneficiaria FONASA & 49.901 & 61.603 & 63.831 & 91.657 & 101.653 \\
\hline & 10.910 .702 & 11.120 .094 & 11.479 .384 & 11.740 .688 & 12.248 .257 \\
\hline Médicos servicios de salud - Cargos & & & Variaciones \% & $\mathbf{2 0 0 6}$ & \\
\hline Médicos servicios de salud - Personas & $\mathbf{2 0 0 4 - 2 0 0 5}$ & $\mathbf{2 0 0 5 - 2 0 0 6}$ & $\mathbf{2 0 0 6 - 2 0 0 7}$ & $\mathbf{2 0 0 7 - 2 0 0 8}$ & $\mathbf{2 0 0 4 - 2 0 0 8}$ \\
\hline Médicos servicios de salud - Horas & 10,03 & 3,40 & 4,11 & 4,99 & 24,37 \\
\hline Médicos APS municipal - Personas & 8,22 & 2,18 & 3,86 & 4,63 & 20,17 \\
\hline Médicos APS municipal - Horas & 7,07 & 2,04 & 3,28 & 4,56 & 17,99 \\
\hline Población beneficiaria FONASA & 16,72 & 10,09 & 30,77 & 8,24 & 81,87 \\
\hline Población total Chile variación 2004-2008 & 23,45 & 3,62 & 43,59 & 10,91 & 103,71 \\
\hline
\end{tabular}

Fuentes: SIRH, Base de datos Atención Primaria de Salud (APS), Fonasa, Instituto Nacional de Estadísticas INE. Incluye a médicos generales y especialistas. 
tir su ejercicio laboral entre el sector público y privado.

El factor principal de crecimiento 2004-2005 de la disponibilidad de horas en los Servicios de Salud ha sido la implementación del Sistema de Garantías en Salud.

La estabilización, entre 2007 y 2008 de la tasa de médicos y horas disponibles en los Servicios de Salud y la Atención Primaria Municipal por 100.000 beneficiarios (Figuras 4 y 5), se debe al incremento de la población beneficiaria de Fonasa a un ritmo tres veces superior al de la población general ( $+6,6 \%$ versus $+2,01 \%$, entre 2006 y 2008 ).

La Figura 6 muestra este efecto poblacional sobre la tasa de horas de médicos disponibles en los Servicios de Salud (excluyendo la APS) por 100.000 beneficiarios del Fonasa.

2.2. Casi la mitad de los médicos contratados en los Servicios de Salud tiene menos de 40 años en 2008 y 21\% menos de 30 (en 2004, éstos últimos representaban sólo el 14\%). Sin embargo, varias
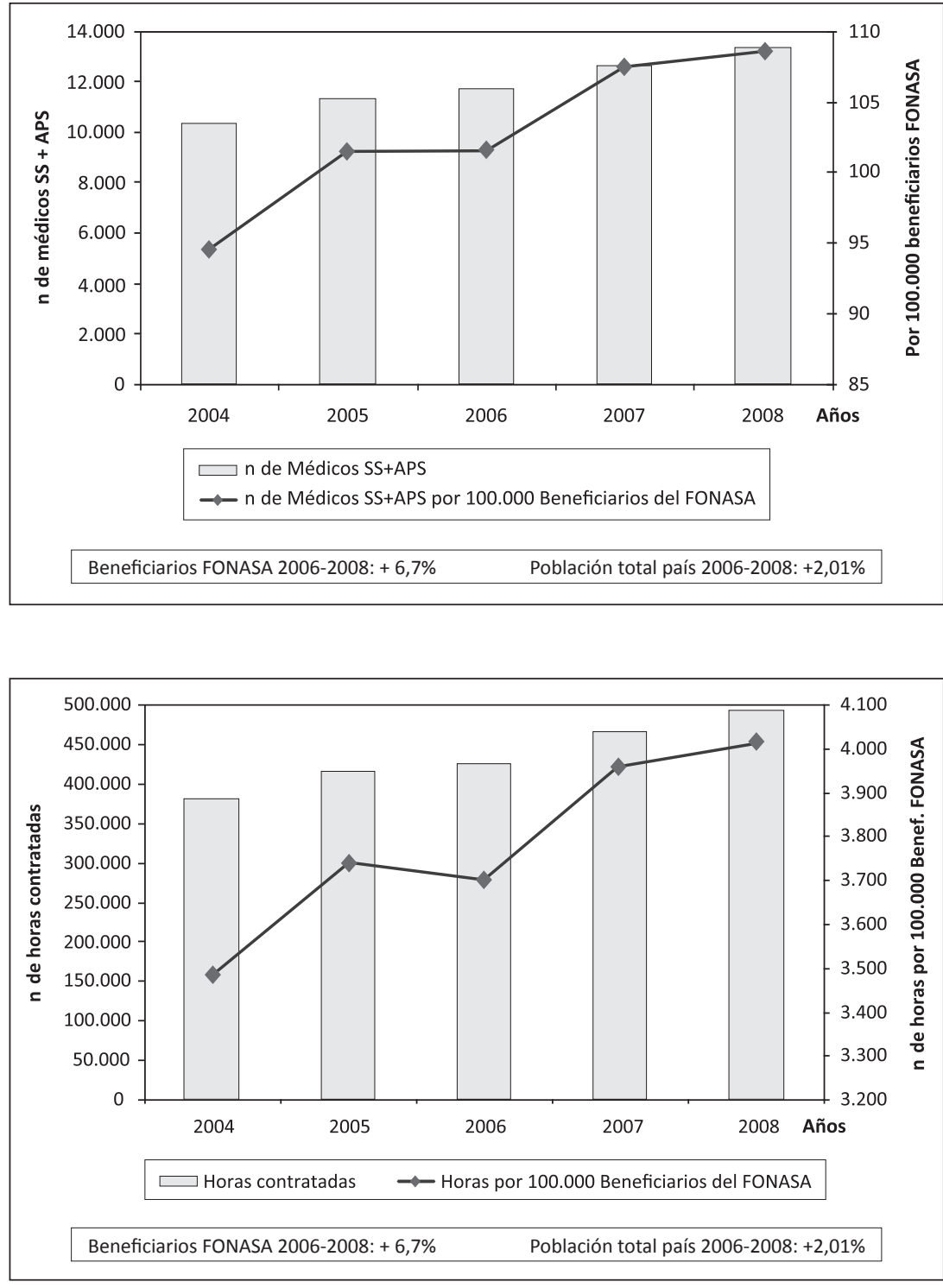

Figura 4. Evolución número de Médicos en Servicios de Salud y Atención Primaria, absoluto y por 100.000 Beneficiarios de Fonasa. Fuentes: SIRH, Base de datos Atención Primaria de Salud (APS), Fonasa, Instituto Nacional de Estadísticas (INE). Incluye a médicos generales y especialistas.
Figura 5. Evolución número de horas médicas contratadas en Servicios de Salud y Atención Primaria, absoluto y por 100.000 Beneficiarios de Fonasa. Fuentes: SIRH, Base de datos Atención Primaria de Salud (APS), Fonasa, Instituto Nacional de Estadísticas INE. Incluye a médicos generales y especialistas. 


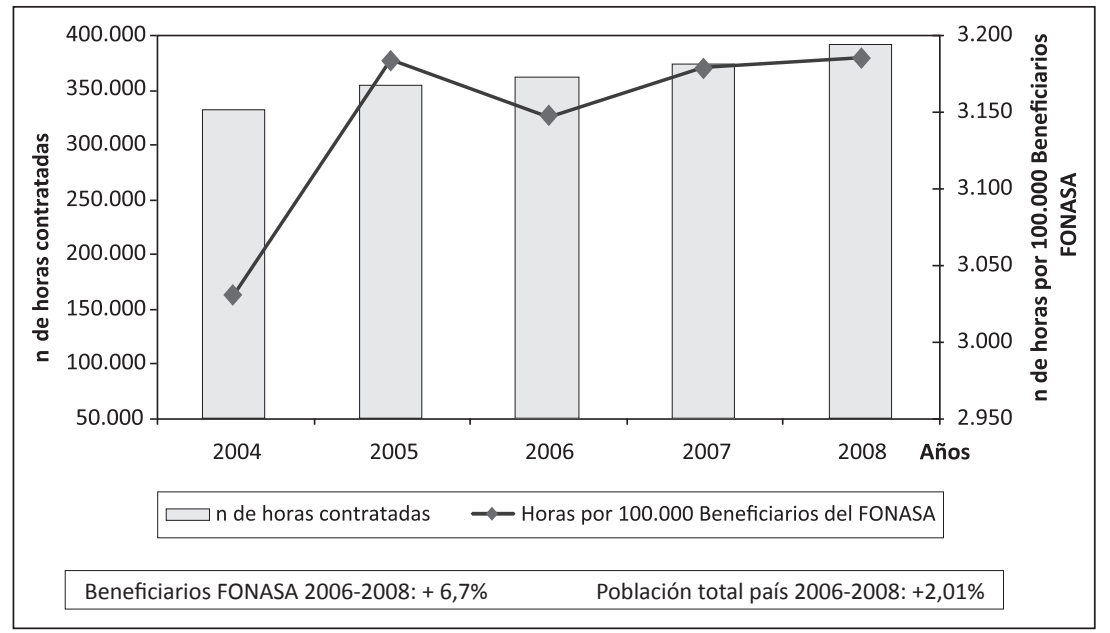

Tabla 3. Estructura etaria de los médicos según la especialidad. Año 2008

\begin{tabular}{|c|c|}
\hline \% Mayores de 50 años & Especialidades \\
\hline Superior o igual a $60 \%$ & $\begin{array}{l}\text { Enfermedades respiratorias } \\
\text { Reumatología } \\
\text { Infectología } \\
\text { Gastroenterología } \\
\text { Diabetología y endocrinología } \\
\text { Hematología y hemato. Onco. Pediátrica }\end{array}$ \\
\hline Entre 50 y $59 \%$ & $\begin{array}{l}\text { Cardiología } \\
\text { Geriatría } \\
\text { Cirugía plástica y reparadora } \\
\text { Nefrología } \\
\text { Cirugía vascular periférica } \\
\text { Obstetricia y ginecología } \\
\text { Medicina intensiva y cuidados intensivos pediátricos } \\
\text { Cirugía de tórax } \\
\text { Inmunología } \\
\text { Medicina nuclear }\end{array}$ \\
\hline Entre 40 y $49 \%$ & $\begin{array}{l}\text { Anatomía patológica } \\
\text { Oncología médica } \\
\text { Pediatría y neonatología } \\
\text { Cirugía pediátrica } \\
\text { Medicina interna } \\
\text { Medicina física y rehabilitación } \\
\text { Otorrinolaringología } \\
\text { Cirugía cardiovascular } \\
\text { Cirugía de cabeza, cuello y plástica máxilo facial } \\
\text { Urología }\end{array}$ \\
\hline Menos de $40 \%$ & $\begin{array}{l}\text { Cirugía general } \\
\text { Neurología adultos y neurología pediátrica } \\
\text { Anestesiología } \\
\text { Dermatología } \\
\text { Neurocirugía } \\
\text { Traumatología y ortopedia } \\
\text { Imagenología } \\
\text { Oftalmología }\end{array}$ \\
\hline
\end{tabular}

Fuente: Estudio Banco Mundial (Referencia 8). 
especialidades tales como enfermedades respiratorias, gastroenterología, cardiología, obstetricia y geriatría, entre otras, incluyen a más de $50 \%$ de médicos mayores de 50 años (Tabla 3), lo que se tiene que considerar en las políticas de formación de especialistas.

\section{Distribución por especialidad}

3.1. De la muestra de 22.822 profesionales públicos y privados identificados a través de la metodología antes señalada (76\% del total de los médicos del país), 15.668 (68,65\%) cumplen funciones de especialidad. En 2008, en los establecimientos públicos dependientes de los Servicios de Salud, los especialistas representaban $81,21 \%$ del total de los médicos contratados (y $64 \%$ del total Servicios de Salud y APS).

De la comparación entre segmento privado y público, no se puede inferir conclusiones definitivas sino tendencias, pues sólo los datos provenientes de los registros del sector público son completos.

Se aprecia la distribución según las principales especialidades en la Tabla 4. Destaca la importancia de las especialidades básicas (más del 47\% del total).

3.2. En la Tabla 5, se puede observar que la tendencia a la concentración en la región Centro varía discretamente según la especialidad. Más de $80 \%$ de los cardiólogos, cirujanos de tórax, oncólogos, neurólogos pediátricos y psiquiatras se encuentran en la región central ( $\mathrm{V}^{\mathrm{a}}, \mathrm{VI}^{\mathrm{a}}, \mathrm{VII}^{\mathrm{a}}$ y XIII ${ }^{a}$ regiones).

Esta diferencia se refleja en la relación médicos especialistas por habitantes por área, tal como se puede constatar en la Tabla 6, donde se nota que la Región Central dispone de 11,15 especialistas por cada 10.000 habitantes, mientras las Regiones Centro Sur, Sur y Norte presentan tasas más bajas.

\section{Discusión}

El conocimiento del universo y de las tendencias en materia de disponibilidad de recursos humanos especializado en Chile, ha alcanzado un nivel de mayor precisión, a través de los distintos estudios realizados por el Ministerio de Salud y el Banco Mundial. Este conocimiento descansa sobre una información que proviene de datos
Tabla 4. Distribución de los médicos por especialidad en una muestra correspondiente a $76 \%$ del universo total público/privado

\begin{tabular}{|lcc|}
\hline Especialidad & \multicolumn{2}{c|}{ Médicos } \\
& Cantidad & \multicolumn{1}{c|}{$\%$} \\
\hline Anatomía patológica & 208 & $1,33 \%$ \\
\hline Anestesiología & 960 & $6,13 \%$ \\
\hline Cardiología & 325 & $2,07 \%$ \\
\hline Cirugía de tórax y cardiovascular & 96 & $0.61 \%$ \\
\hline Cirugía general & 2.135 & $13,63 \%$ \\
\hline Cirugía pediátrica & 381 & $2,43 \%$ \\
\hline Dermatología & 311 & $1,98 \%$ \\
\hline Imagenología & 486 & $3,10 \%$ \\
\hline Medicina física y rehabilitación & 118 & $0,75 \%$ \\
\hline Medicina interna & 1.717 & $10,96 \%$ \\
\hline Medicina nuclear & 29 & $0,19 \%$ \\
\hline Neurocirugía & 204 & $1,30 \%$ \\
\hline Neurología & 354 & $2,26 \%$ \\
\hline Neurología pediátrica & 68 & $0,43 \%$ \\
\hline Obstetricia y ginecología & 1.638 & $10,45 \%$ \\
\hline Oftalmología & 667 & $4,26 \%$ \\
\hline Oncología médica & 62 & $0,40 \%$ \\
\hline Ortopedia y traumatología & 849 & $5,42 \%$ \\
\hline Otorrinolaringología & 382 & $2,44 \%$ \\
\hline Pediatría & 1.882 & $12,01 \%$ \\
\hline Psiquiatría & 735 & $4,69 \%$ \\
\hline Urología & 323 & $2,06 \%$ \\
\hline Otras especialidades & 1.738 & $11,09 \%$ \\
\hline Total & 15.668 & $100 \%$ \\
\hline Eente: Estudio Banco & & \\
\hline
\end{tabular}

Fuente: Estudio Banco Mundial (Referencia 8). Nota: La diferencia entre el total de la tabla No 4 (15.668) y la muestra estudiada (22.822) corresponde a los Médicos Generales (7.154).

reales registrados en diferentes bases de datos, $\mathrm{y}$ no en proyecciones. Sin perjuicio de lo anterior, es necesario señalar dos limitaciones en la aplicación de la metodología:

- La información entregada por el Servicio de Impuestos Internos sólo incluye a los médicos que tributan en forma individual.

- Se logró identificar la especialidad de $76 \%$ de los médicos registrados en el Servicio de Impuestos Internos. 
Tabla 5. Distribución geográfica de los médicos de la muestra, por especialidad

\begin{tabular}{|c|c|c|c|c|c|}
\hline \multirow[t]{2}{*}{ Especialidad } & \multicolumn{4}{|c|}{ Áreas geográficas } & \multirow{2}{*}{$\begin{array}{c}\text { Total áreas } \\
\text { (\%) }\end{array}$} \\
\hline & $\begin{array}{c}\text { Norte } \\
(\%)\end{array}$ & $\begin{array}{c}\text { Central } \\
(\%)\end{array}$ & $\begin{array}{c}\text { Centro-sur } \\
(\%)\end{array}$ & $\begin{array}{l}\text { Sur } \\
(\%)\end{array}$ & \\
\hline Anatomía patológica & 9,13 & 72,12 & 12,98 & 5,77 & 100,00 \\
\hline Anestesiología & 6,56 & 74,69 & 14,27 & 4,48 & 100,00 \\
\hline Cardiología & 5,23 & 81,23 & 11,38 & 2,15 & 100,00 \\
\hline Cirugía de tórax y cardiovascular & 1,04 & 85,42 & 12,50 & 1,04 & 100,00 \\
\hline Cirugía general & 13,11 & 67,68 & 13,49 & 5,71 & 100,00 \\
\hline Cirugía pediátrica & 3,94 & 77,17 & 15,49 & 3,41 & 100,00 \\
\hline Dermatología & 7,40 & 75,88 & 11,90 & 4,82 & 100,00 \\
\hline Imagenología & 7,41 & 72,02 & 15,43 & 5,14 & 100,00 \\
\hline Medicina física y rehabilitación & 5,93 & 77,12 & 13,56 & 3,39 & 100,00 \\
\hline Medicina interna & 8,27 & 71,75 & 15,49 & 4,48 & 100,00 \\
\hline Medicina nuclear & 3,45 & 72,41 & 20,69 & 3,45 & 100,00 \\
\hline Neurocirugía & 8,82 & 71,08 & 15,69 & 4,41 & 100,00 \\
\hline Neurología & 4,80 & 74,29 & 15,54 & 5,37 & 100,00 \\
\hline Neurología pediátrica & 1,47 & 91,18 & 4,41 & 2,94 & 100,00 \\
\hline Obstetricia y ginecología & 7,14 & 73,87 & 14,29 & 4,70 & 100,00 \\
\hline Oftalmología & 8,10 & 71,81 & 15,89 & 4,20 & 100,00 \\
\hline Oncología médica & 1,61 & 82,26 & 16,13 & 0,00 & 100,00 \\
\hline Ortopedia y traumatología & 6,36 & 75,74 & 13,19 & 4,71 & 100,00 \\
\hline Otorrinolaringología & 8,90 & 69,63 & 16,75 & 4,71 & 100,00 \\
\hline Pediatría & 9,78 & 66,21 & 18,23 & 5,79 & 100,00 \\
\hline Psiquiatría & 4,22 & 80,00 & 12,65 & 3,13 & 100,00 \\
\hline Urología & 9,91 & 72,45 & 12,69 & 4,95 & 100,00 \\
\hline Otras especialidades & 2,99 & 82,97 & 11,91 & 2,13 & 100,00 \\
\hline Total & 7,32 & 73,47 & 14,42 & 4,45 & 100,00 \\
\hline
\end{tabular}

Fuente: Estudio Banco Mundial (Referencia 8). Nota: Norte: Regiones I, II, III, IV y XV, Central: Regiones V, VI, VII y XIII, Centro Sur: Regiones VIII, IX y XIV, Sur: Regiones X, XI y XII.

Es necesario consolidar estos avances, aumentando la información sobre el sector privado, donde el Registro de la Superintendencia de Salud aportará una mejoría significativa.

También se requiere definir metodologías y desarrollar información para conocer el potencial de atención que representa un determinado número de especialistas, en horas y prestaciones, lo que permitirá estimar de mejor forma la cantidad de recursos humanos que se requiere para atender la demanda de atención especializada actual y futura.
De los resultados presentados, se pueden destacar características relevantes para orientar decisiones de políticas respecto de la disponibilidad y distribución de personal especializado.

- Los médicos públicos y privados se concentran en la zona central del país, lo que expresa inequidad en el acceso de la población a la atención de Salud. Las diferencias en el número de profesionales por habitantes es particularmente marcada en algunas especialidades tales como anestesiología, cardiología y psiquiatría. 
Tabla 6. Número de médicos especialistas de la muestra, por cada 10.000 habitantes, por área geográfica y especialidad

\begin{tabular}{|c|c|c|c|c|c|}
\hline \multirow[t]{2}{*}{ Especialidad } & \multicolumn{4}{|c|}{ Áreas geográficas } & \multirow{2}{*}{$\begin{array}{c}\text { Total áreas } \\
\text { (\%) }\end{array}$} \\
\hline & $\begin{array}{c}\text { Norte } \\
(\%)\end{array}$ & $\begin{array}{c}\text { Central } \\
(\%)\end{array}$ & $\begin{array}{c}\text { Centro-sur } \\
(\%)\end{array}$ & $\begin{array}{l}\text { Sur } \\
(\%)\end{array}$ & \\
\hline Anatomía patológica & 0,1 & 0,15 & 0,08 & 0,11 & 0,13 \\
\hline Anestesiología & 0,32 & 0,7 & 0,41 & 0,4 & 0,58 \\
\hline Cardiología & 0,09 & 0,26 & 0,11 & 0,07 & 0,2 \\
\hline Cirugía de tórax y cardiovascular & 0,01 & 0,08 & 0,04 & 0,01 & 0,06 \\
\hline Cirugía general & 1,4 & 1,41 & 0,87 & 1,15 & 1,29 \\
\hline Cirugía pediátrica & 0,08 & 0,29 & 0,18 & 0,12 & 0,23 \\
\hline Dermatología & 0,12 & 0,23 & 0,11 & 0,14 & 0,19 \\
\hline Imagenología & 0,18 & 0,34 & 0,23 & 0,24 & 0,29 \\
\hline Medicina física y rehabilitación & 0,04 & 0,09 & 0,05 & 0,04 & 0,07 \\
\hline Medicina interna & 0,71 & 1,21 & 0,8 & 0,72 & 1,03 \\
\hline Medicina nuclear & 0,01 & 0,02 & 0,02 & 0,01 & 0,02 \\
\hline Neurocirugía & 0,09 & 0,14 & 0,1 & 0,08 & 0,12 \\
\hline Neurología & 0,09 & 0,26 & 0,17 & 0,18 & 0,21 \\
\hline Neurología pediátrica & 0,01 & 0,06 & 0,01 & 0,02 & 0,04 \\
\hline Obstetricia y ginecología & 0,59 & 1,18 & 0,71 & 0,72 & 0,99 \\
\hline Oftalmología & 0,27 & 0,47 & 0,32 & 0,26 & 0,4 \\
\hline Oncología médica & 0,01 & 0,05 & 0,03 & 0,00 & 0,04 \\
\hline Ortopedia y traumatología & 0,27 & 0,63 & 0,34 & 0,38 & 0,51 \\
\hline Otorrinolaringología & 0,17 & 0,26 & 0,19 & 0,17 & 0,23 \\
\hline Pediatría & 0,92 & 1,22 & 1,03 & 1,02 & 1,13 \\
\hline Psiquiatría & 0,16 & 0,58 & 0,28 & 0,22 & 0,44 \\
\hline Urología & 0,16 & 0,23 & 0,12 & 0,15 & 0,19 \\
\hline Otras especialidades & 0,26 & 1,41 & 0,62 & 0,35 & 1,05 \\
\hline Total & 5,92 & 11,15 & 6,77 & 6,49 & 9,35 \\
\hline
\end{tabular}

Fuente: Estudio Banco Mundial (Referencia 8). Nota: Norte: Regiones I, II, III, IV y XV, Central: Regiones V, VI, VII y XIII, Centro Sur: Regiones VIII, IX y XIV, Sur: Regiones X, XI y XII.

El número de habitantes por médico presenta diferencias notables según el tipo de seguros al que la población se encuentra afiliada.

- El aumento sostenido de las expansiones de horas médicas especializadas en los Servicios de Salud, entre 2004 y 2008, ha permitido mantener el número de horas por 100.000 beneficiarios, a pesar del crecimiento importante de la población beneficiaria de Fonasa.

- Los Servicios de Salud cuentan con profesionales especializados cada vez más jóvenes. Sin embargo, el alto porcentaje de médicos de más de 50 años en algunas especialidades es un factor a considerar en la planificación de la formación.

- Los resultados presentados muestran que revertir la tendencia a la concentración de los médicos especializados en algunas regiones, es uno de los desafíos más críticos que debe enfrentar una política de recursos humanos orientada a lograr una mayor equidad en el acceso de la población. 
Contar con conocimiento sobre los factores que condicionan la oferta y demanda de los profesionales, es indispensable para desarrollar una planificación moderna, entendida como el "proceso continuo y dinámico de concertación social con los actores relevantes, orientado a la toma de decisión, y que apunta a prever los recursos requeridos para lograr determinados objetivos, según un orden de prioridad establecido" 14 , facilitando el fortalecimiento de la red asistencial y el logro de los objetivos sanitarios.

En esta perspectiva, el Ministerio de Salud está implementando un sistema de trabajo ${ }^{15}$, que, a partir de un modelo informatizado diseñado en el marco del Estudio Banco Mundial-Gobierno de Chile, permitirá estimar brechas de recursos humanos, comenzando por los médicos especialistas, en base a la construcción de estándares nacionales o de la utilización de valores referenciales existentes, considerados como metas a alcanzar en un determinado período. Este modelo y los primeros resultados de su aplicación serán motivo de una futura publicación.

\section{Referencias}

1. Comisión de las Comunidades Europeas. Libro Verde sobre el personal sanitario europeo. Promover la sostenibilidad del personal sanitario europeo. Bruselas COM (2008) 725.

2. Brito P, Padilla M, Rigoli F. Planificación de recursos humanos y reformas del sector salud. Rev Cubana Educ Med Super 2002; 16(4).

3. Dussault G, Rigoli F. The Interface between Sector Reform and Human Resources in Health. Health, Nutrition and Population (HNP). Discussion Paper. World Bank; 2003.

4. Organización Panamericana de la Salud. Llamado a la acción de Toronto. 2006-2015: Hacia una década de Recursos Humanos en Salud para las Américas. Reunión Regional de los Observatorios de Recursos Humanos en Salud. 4-7 de octubre de 2005.

5. Guillou M. La atención médica especializada en el sector público de salud: estimación de las necesidades y definición de políticas. En: Organización Panamericana de la Salud. Ministerio de Salud de Chile, editores. El Desarrollo del Personal Sanitario en los Gobiernos Democráticos 1990-2008. Antecedentes y aportes de actores relevantes. Santiago, Chile; 2008. p. 61-76.

6. Ministerio de Salud. Informe de Análisis de Brechas de Atención Médica Especializada en el Sistema Nacional de Servicios de Salud. Santiago de Chile: Ministerio de Salud; 2007.

7. Ministerio de Salud. Análisis de la tasa de contratación en los Servicios de Salud, de los médicos becados por el Ministerio de Salud para especializarse: cohorte $1997-$ 2008. Santiago de Chile: Ministerio de Salud; 2008.

8. Ministerio de Salud, Banco Mundial. Estudio de brechas de oferta y demanda de Médicos Especialistas en Chile. Colaboración técnica del Banco Mundial y el Gobierno de Chile. Informe final. Buenos Aires: Banco Mundial; 2009.

9. Deliege D, Artoisenet C. Les médecins dans 23 pays industrialisés. Densité et place des spécialistes. Louvain: Université Catholique. Unité de Socio- Economie de la Santé; 2005.

10. Council of Graduate Medical Educación. Evaluation of Specialty Physician Workforce Methodologies. Resource Paper. New York: US Department of Health and Human Services. Health Resources and Services Administration; 2000.

11. Council of Graduate Medical Educación. Physician Workforce Policy Guidelines for the United States, 2000 2020. New York: US Department of Health and Human Services. Health Resources and Services Administration; 2005. 16th Report.

12. Subsecretaria de Redes Asistenciales. Ministerio de Salud. Actualización de datos y campos de la hoja de vida del personal contratado bajo las leyes 15.076, 19.664 y honorarios, en el Sistema Nacional de Servicios de Salud. Orientaciones técnicas. Santiago de Chile: Ministerio de Salud; 2009.

13. Bastías G, Marshall G, Zúñiga D, Beltrán M. Número de Médicos en Chile: estimaciones, proyecciones y comparación internacional. Rev Med Chile 2000; 128 : 1167-76.

14. Jourdain A, Frossard M, Editores. Les nouveaux outils de la planification sanitaire. Actualités et dossier en Santé Publique. Paris : Haut Conseil en Santé Publique; Juin 1995. http://www.hcsp.fr/explore.cgi/adsp?ae=adsp\&cle $\mathrm{f}=33 \& \mathrm{menu}=111282$. Consultado el 26 de noviembre de 2009.

15. Subsecretaria de Redes Asistenciales. Ministerio de Salud. Estudio de Brecha de Oferta y Demanda de Médicos Especialistas. Cuaderno de Redes $\mathrm{N}$ o 31; enero de 2010 . 


\section{Anexo 1. Principales fuentes de información sobre los médicos en Chile}

\begin{tabular}{|c|c|}
\hline Fuente & Principales contenidos accesibles \\
\hline 1. Servicio de Impuestos Internos & Número de médicos por comuna y tramo de renta (si > 10). \\
\hline 2. Conacem & $\begin{array}{l}\text { Médicos certificados, identificados por RUT, especialidad, y comuna } \\
\text { (excepto Región Metropolitana) }\end{array}$ \\
\hline 3. Fonasa & $\begin{array}{l}\text { Médicos con convenio vigente de atención por modalidad de libre } \\
\text { elección, identificados por RUT, especialidad y comuna }\end{array}$ \\
\hline 4. Base de datos del desempeño difícil APS & $\begin{array}{l}\text { Médicos contratados en la APS municipal, identificados por RUT, fecha } \\
\text { de nacimiento, sexo, nacionalidad, cargo, datos de remuneración, } \\
\text { establecimiento de desempeño y comuna }\end{array}$ \\
\hline $\begin{array}{l}\text { 5. Sistema de Información Integrado de Recursos } \\
\text { Humanos (Sector Público: Servicios de Salud) }\end{array}$ & $\begin{array}{l}\text { Médicos contratados en los Servicios de Salud, identificados por RUT, } \\
\text { fecha de nacimiento, sexo, nacionalidad, especialidad, cargo, datos de } \\
\text { remuneración, establecimiento y comuna }\end{array}$ \\
\hline $\begin{array}{l}\text { 6. Ciclo de destinación y formación del Ministerio } \\
\text { de Salud }\end{array}$ & $\begin{array}{l}\text { Médicos becados por el Ministerio de Salud, identificados por RUT, } \\
\text { establecimiento de origen y lugar de formación, años de inicio y } \\
\text { término de la beca }\end{array}$ \\
\hline $\begin{array}{l}\text { 7. Registro Nacional de Prestadores Individuales de } \\
\text { Salud, mantenido por la Superintendencia de } \\
\text { Salud* }\end{array}$ & $\begin{array}{l}\text { Médicos identificados por RUT, fecha de nacimiento, sexo, nacionali- } \\
\text { dad, titulo profesional, especialidad, centro formador que otorga el } \\
\text { título, origen de la certificación, lugar de trabajo e información de } \\
\text { contacto }\end{array}$ \\
\hline
\end{tabular}

*A la fecha de redacción del artículo, este registro cuenta con un buen nivel de poblamiento en la profesión médica y sus especialidades.

\section{Anexo 2. Proceso de identificación de los médicos en Chile}

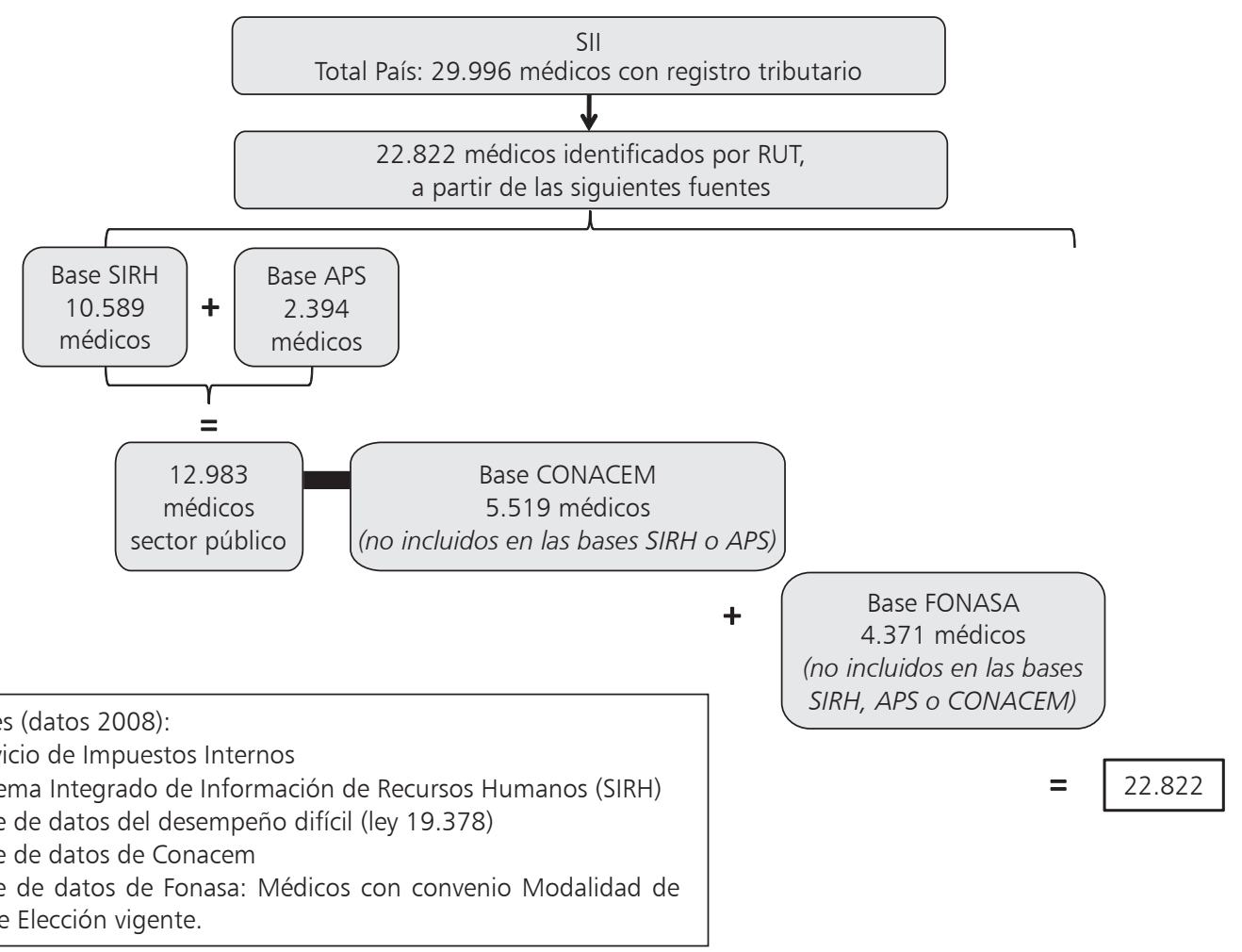

